\title{
Condition assessment for the performance degradation of bearing based on a combinatorial feature extraction method
}

\author{
Sheng Hong ${ }^{\mathrm{a}}$, Zheng Zhou ${ }^{\mathrm{b}}$, Enrico Zio ${ }^{\mathrm{c}, \mathrm{d}}$, Kan Hong ${ }^{\mathrm{a}, *}$ \\ a Science \& Technology Laboratory on Reliability E Environmental Engineering, School of Reliability and System Engineering, Beihang University, \\ 631 Weimin Building, 37 Xueyuan Rd, Haidian District, Beijing, China \\ b Systems Engineering Research Institute, China State Shipbuilding Corporation (CSSC), China \\ c Department of Energy Polytechnic of Milan, Via Ponzio 34/3, 20133 Milan, Italy \\ ${ }^{d}$ Ecole Central Paris et Supelec, Paris, France
}

Available online 8 January 2014

\section{Introduction}

Bearings are the widely used mechanical parts in rotational equipment and usually constitute a large portion of the failure accidents. In order to avoid the fatal breakdowns of the machines, the bearing defects should be detected as early as possible to pre-vent unexpected failure [1]. Condition based maintenance (CBM), aiming to reduce the cost of maintenance and improve the relia-bility, becomes an efficient strategy during these years. Prognostics of bearing has a great significance to identify the future conditions for the maintenance plans, and also benefit to reduce the produc-tion downtime, the maintenance cost, and the safety hazards [2].

Effective performance degradation assessment is still a challenging problem in academia and industries [3]. Three main approaches, including model-based, data-driven and hybrid prognostics with statistics, are commonly used [4]. The data-driven methodology is directly based on the acquired vibration signals. Although the bearing vibration signals contain very specific infor-mation about the bearing's fault conditions, it's quite difficult to detect and track the weak signals at an early stage [5]. Thus, one of the main challenges in prognostics of the bearing is how to extract the features and construct the proper health indicators from the monitoring signals.

* Corresponding author.

E-mail address: hongminle@163.com (K. Hong).
As the vibration signals of the bearing possess non-stationary characteristic and have the weak faulty signals with strong background noise, the time-frequency methods are considered to be an effective way for extracting the features of the original data [6,7]. The algorithm based on wavelet packet decomposition (WPD) has been applied for the vibration signal analysis [8,9]. WPD, which is capable of dividing the whole time-frequency signal into many dif-ferent frequency bandwidths, has received widespread attentions in these years. The energy of the wavelet packet is commonly used to identify the failure mode of the bearings [10]. Wavelet packet energy entropy, which reflects the energy change of each sub-band when the degradation occurs, is also proved to be a good recognition method in bearing fault diagnosis [11]. In recent years, another time-frequency analysis method named as empirical mode decomposition (EMD) has been more and more widely used in signal processing [12]. The signal will be decomposed into a set of intrinsic mode functions (IMF) that involves both sam-pling frequency and changes with the signal itself. By analyzing each IMF, the feature information of the original signal could be extracted more effectively and accurately. Some successful fault diagnosis methods based on EMD have been carried on which use energy entropy or Hilbert transform to identify the work condi-tion and the fault patterns [13-16]. To overcome the shortcomings in EMD, an improved EMD method using Wavelet Packet Transform (WPT-EMD) are presented in fault diagnosis [17-19].The main purpose of using WPEMD method in those literatures is to 
get more accurate bearing fault characteristic information. WPD is used for de-noising the signal and then put the signal decomposed into each IMFs component for envelope analysis or energy characteristics extraction. Q. Liu estimated the fault frequency by using Wavelet Package Transform and Ensemble Empirical Mode Decomposition [20]. A. Santhana Raj and N. Murali used Morlet wavelet Undecimated Discrete Wavelet Transform (UDWT) de-noising and EMD method to find the characteristic frequencies of the bearing fault [21]. However, in those methods, the trend information of IMFs is not being fully utilized and single fault frequency is not enough for degradation trend analysis. More proper features for trend analysis still need to be constructed.

The progressions of those vibration based features are not monotonic during the degradation of the bearing, which makes the remaining useful life (RUL) prediction even more challenging. Health indicator is introduced for evaluating the performance of the bearing from available features. Different features are sensitive to different faults and degradation severity [22]. A proper health indicator should be applied to describe the whole progression of the degradation for condition assessment. And this is very dif-ferent from the traditional failure diagnosis which basically just needs to identify different failure modes [23]. Caesarendra et al. proposed a combination method using logistic regression (LR) and relevance vector machine (RVM) to assess the state of health and predict the RUL [24]. Huang et al. estimated the degradation con-dition and the RUL by using a selforganizing map (SOM) and back propagation (BP) neural network [25]. Zhao Wei et al. utilized a dynamic particle filter-support vector regression method for relia-bility prediction [26]. With each claiming the effectiveness of the proposed method based on the experimental results on a certain data set, those methods still have some shortcomings when multiple faults occur. Usually, a specific fault data is used for training the model and a fairly good result can be obtained in diagnosis. However, it is quite impossible for us to know the specific failure mode that will occur in a real-world prognosis, especially for a long-term prediction with multiple faults occurrences. Thus, another main challenge in the bearing prognostics is how to provide an effective condition assessment to describe the development of the performance degradation and make an accurate RUL prediction for proactive maintenance.

To solve problems mentioned above, this paper presents a condition assessment approach based on the degraded signal. The main contribution concerns the utilization of the WPD and EMD technique to extract features, as well as SOM neural networks to construct health indicators achieving the performance degradation assessment and the RUL estimation. The original signals are decomposed by the WPD and the corresponding entropy features are extracted from the time series that are obtained after the wavelet coefficient reconstruction. EMD, served as a trend analysis method, is applied to extract the entropy sequences which are taken as the input vectors of the SOM network. The confidence value (CV) de-rived from the SOM are used to illustrate the health state and estimate the RUL. To verify the superiority of the proposed method, it is compared with some traditional features. Seventeen datasets from a bearing run-to-failure test are used for assessment as well. The experimental results show that the prognostic approach of the proposed method can be severed as a powerful way to describe the evolution of the bearing degradation.

This paper is organized as follows. In Section 2, the combinatorial feature extraction method of WPD and EMD are presented. In Section 3, the proposed assessment method is described in details. Section 4 presents the results of the proposed method on the condition assessment of the performance degradation and the RUL estimation using the experiment signals. General conclusions are presented in Section 5.

\section{Combination feature extraction based on WP-EMD}

In the WPD analysis, the signal is filtered with the low-pass $\left\{h_{k}\right\}$ and the high-pass filters $\left\{g_{k}\right\}$ at first. Thus, WPD can decompose the full frequency-band of the signal into different frequency sub-bands and allow better time-frequency localization of the signals. For a signal $f(t)=x_{0}^{1}(t)$, the decomposition is defined as,

$\left\{\begin{array}{l}x_{j}^{2 i}=\sum_{k} h_{k}(k-2 t) x_{j-1}^{i}(t) \\ x_{j}^{2 i+1}=\sum_{k} g_{k}(k-2 t) x_{j-1}^{i}(t)\end{array}\right.$

The reconstruction process of the $i$-th wavelet on the $j$-th layer is defined as

$x_{j}^{i}(t)=2\left[\sum_{k} h(t-2 k) x_{j+1}^{2 i-1}(t)+\sum_{k} g(t-2 k) x_{j+1}^{2 i}(t)\right]$

where $h(n)$ is the low-pass filter related to the scaling function and $g(n)$ is the high-pass filter related to the wavelet function.

The combination feature extraction uses 3-layers WPD as a preprocessor to de-noise and decompose of the collected vibration signal. Eight waveforms are obtained and designated as $x_{3}^{1}(t) x_{3}^{2}(t) \cdots x_{3}^{8}(t)$. The wavelet energy is varying over different scales depending on the input signals. We use the energy entropy to describe the change of the energy distribution. The energy and the entropy of each sub-band can be calculated as follows,

$E_{j}^{i}(t)=\sum_{n=1}^{N}\left(x_{j}^{i}(t)^{2}\right)$

$S_{\text {en }}=-\sum_{i=1}^{N} p_{j}^{i} \log p_{j}^{i} \quad\left(S_{\text {en }}=0\right.$ when $\left.p_{j}^{i}=0\right)$

where $i, j$ is the number of the wavelet coefficients, $N$ is the length of the signal $x_{j}^{i}(t)$ and $p_{j}^{i}$ is the energy probability distribution of the signal on the $j$-th layer, which is defined as

$p_{j}^{i}=\frac{E_{j}^{i}(t)}{\sum_{j} E_{j}^{i}(t)}$

After calculate each entropy of the collected vibration signals, the entropy sequences $s_{e n}(t)$ in whole life time series can be obtained. As bearing operating, the entropy sequences will be degraded. To analyze the evolution of degradation more concisely, EMD is applied to extract the trend of entropy sequences.

EMD is a self-adaptive method to decompose nonlinear and non-stationary signals developed by Huang in 1998 [27]. It decomposes the signal into a number of IMFs and the residue of the decomposition. Given an input signal $x(t)$, the EMD procedure can be express as the following formulas,

$\left\{\begin{array}{l}m(t)=\left(e_{\max }(t)+e_{\min }(t)\right) / 2 \\ c_{i}(t)=x(t)-m_{i}(t) \\ r_{i}(t)=x(t)-c_{i}(t)\end{array}\right.$

where $e_{\max }(t)$ and $e_{\min }(t)$ are the upper envelope and the lower envelope obtained from all the local maxima of the signal $x(t)$ using a cubic spline line. Then, take the residual $r_{1}(t)$ as the original signal and iterate (6). As a result, $n$ IMFs can be obtained as follows, 


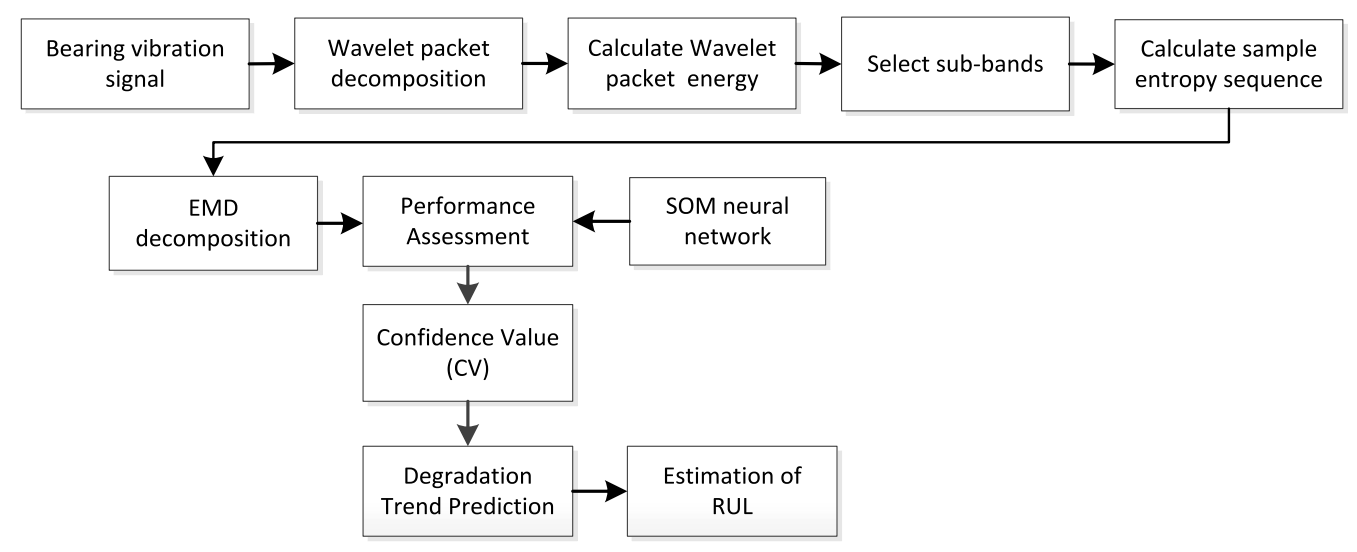

Fig. 1. Overview of the condition assessment method.

$r_{2}(t)=r_{1}(t)-c_{2}(t)$

$r_{3}(t)=r_{2}(t)-c_{3}(t)$

$\cdots$

$r_{n}(t)=r_{n-1}(t)-c_{n}(t)$

In practice, the above procedure will repeat until the residual item $r_{n}(t)$ becomes a monotonic function or the IMF $c_{n}(t)$ can be considered as zero-mean signal. Finally, the original signal $x(t)$ is decomposed into $n$-empirical modes as follows,

$x(t)=\sum_{i=1}^{n} c_{i}(t)+r_{n}(t)$

where $r_{n}(t)$, the residual function shows the average trend of the signal and the IMFs $c_{i}(t)$, include different frequency bands of the signal from high frequency to low frequency.

We can achieve a decomposition of the entropy sequences $s_{e n}(t)$ into $n$-empirical modes using (8). Generally, the first of several major IMFs are considered to contain the faulty information and can be adopted to pattern recognition by using some classification algorithm like SVM, which have been reported in many studies. But it may be not enough for us to distinguish the fault pattern due to the lack of learning data and the occurrence of multiple faults. The last few IMFs are often ignored in traditional diagnosis methods. However, those IMFs illustrate the tendency of the origin signal that can be used for illustrating the change of the signal. To describe the entropy sequences trend precisely, we define the entropy sequences trend $s_{\text {Trend }}(t)$ of the selected sub-bands by considering last $L$ IMFs and then construct the feature vectors $\boldsymbol{T}$ :

$s_{\text {Trend }}(t)=\sum_{i=L}^{n} c_{i}(t)+r(t)$

$\boldsymbol{T}=\left[\begin{array}{llll}s_{\text {Trend }_{1}}(t) & s_{\text {Trend }_{2}}(t) & \cdots & s_{\text {Trend }_{n}}(t)\end{array}\right]$

\section{Condition assessment method}

In this section, the main steps of the proposed method are presented. Schematic diagram of the proposed model is shown in Fig. 1.

Performance condition can be assessed according to the feature vectors from the monitoring signal. We use the $\mathrm{CV}$ as a health indicator which represents the state of bearing by using a number between 0 and 1 [28]. A higher CV represents a normal state, whereas a lower CV indicates a failure state. Four stages are classified as normal, slight degradation, severely degradation and failure. Each threshold should be depended on the actual needs. Thus, different maintenance strategy can be carried on according to the different stages.

The CV is obtained from the SOM neural network, which is a non-supervised learning neural network designed to organize itself according to the input data. The map units of SOM form a two-dimensional topology on the output space. The number of map units is approximately $d=5 \sqrt{k}$, where $k$ is the number of data samples. Each neuron $i$ in the SOM is represented by an $n$-dimensional weight vector $m_{i}=\left[m_{i 1}, m_{i 2}, \ldots, m_{i n}\right]$, where $n$ is the dimension of the input vectors.

The SOM is trained by the feature vectors under the normal condition first. Then, as the bearing operates, the feature vectors obtained by the vibration signal are calculated to the trained SOM incessantly. For an input vector $\boldsymbol{T}$, the minimum quantization error (MQE) is used to quantize the degradation condition.

$M Q E=\left\|\boldsymbol{T}-\boldsymbol{m}_{B M U}\right\|$

where $\boldsymbol{T}$ is the input data vector and $\boldsymbol{m}_{\boldsymbol{B M U}}$ stands for the weight vector of Best Matching Unit (BMU) of the input $\boldsymbol{T}$ [29]. Then, the $\mathrm{CV}$ representing the performance of a rolling bearing, can be formulated as follow,

$C V=\frac{c}{\sqrt{M Q E}+c}$

where $c$ is scale parameter determined by the MQE under the normal condition, and $x_{M Q E}$ is the MQE obtained from the monitoring feature vectors.

This assessment method develops a degradation trend-dependent approach for the RUL estimation that allows the estimated RUL to be dependent on the history degradation data and to be dynamic updated when a newly observed data is available. We can start the estimation once the bearing enters the degradation stage and continuously monitor the CV trend.

The RUL estimation approach can be divided into two steps, as depicted in Fig. 2. The first step is an off-line model, which utilizes the signal data under normal state for training the SOM. As more feature vectors are calculated for testing, the progression of bearing degradation can be described by CV curve. The second step is on-line prediction. The health state and the RUL of the bearing will be dynamic updated when the new collected data are available by using the trained SOM in the off-line model. Regression algorithm, such as AR model and SVR, can be applied for the RUL estimation according to the bearing's $\mathrm{CV}$. 


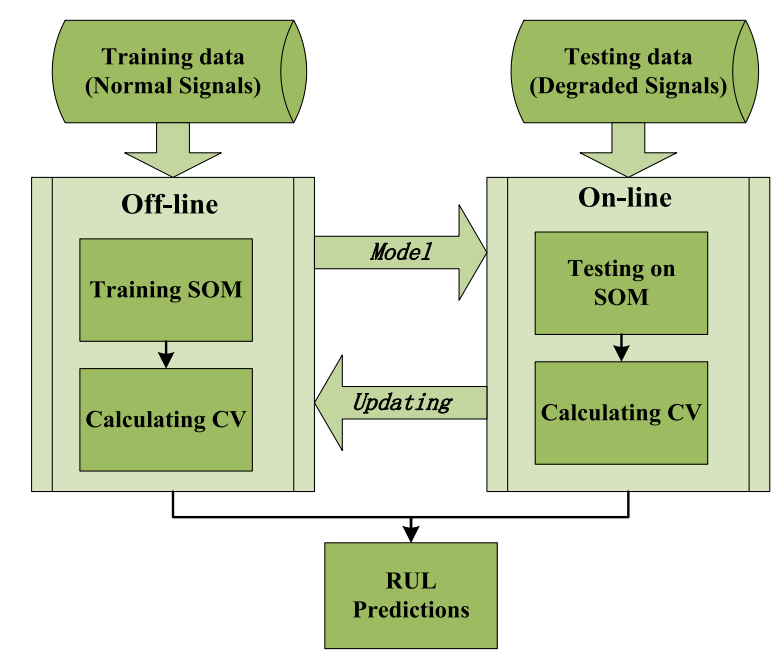

Fig. 2. Framework of the RUL estimation approach.

\section{Experiment analysis and discussion}

\subsection{Description of the experiment}

In order to explain the achieving process and the feasibility of the proposed prediction method, a bearing run-to-failure test data is taken as a case study. The bearing test dataset was generated and collected from the bearing Accelerated Life Tests (ALT) of 17 bearings [30]. The bearing experimentation platform is depicted in Fig. 3. The vibration data for each bearing include two channels from two accelerometers placed radially on the external race of the bearing in vertical and horizontal directions, respectively. The load is applied to the bearing radially in horizontal direction. The vibration signal data are collected at a sampling frequency of $25.6 \mathrm{kHz}$ every $10 \mathrm{~s}$ for a period of $0.1 \mathrm{~s}$. Failure of the bearing is claimed when the acceleration amplitude of the vibration signal exceeds the threshold of $20 \mathrm{~g}$. 17 bearings are tested in three different operation condition, as shown in Table 1 . Bearing 1-1, 1-2, 2-1, 2-2, $3-1,3-2$ are six learning data, and the remaining 11 data are used for testing.

\subsection{Condition assessment}

The original acceleration vibration signal of Bearing 1-3, which is the 3rd bearing of the dataset 1 , is presented in Fig. 4. Some commonly used time-domain features of bearing, namely RMS, kurtosis, crest factor and entropy, are calculated as shown in Fig. 5. It can be seen form Fig. 5(a), the change rate of RMS increase significantly at the end of this experiment. The changes of kurtosis and crest factor have similar trends but with more background noise in Fig. 5(b) and Fig. 5(c). For the entropy of this bearing, there is no significant change during the experiment as in Fig. 5(d). Those figures show that the cumulative damage consumes most of the bearing fatigue time and may result in a sudden crack in a rel-atively short time period. Prognosis based on those features cannot be able to provide timely maintenance suggestion. Therefore, we not only can't assess bearing future's performance, but also can hardly estimate the RUL according to those parameter's trend or values. None of them can be used as an effective indicator for the bearing degradation assessment.

In this application, by applying three layers WPD to the original signal with Daubechies 5 ( Db 5) wavelet base, eight time series are reconstructed according to the coefficients of the frequency bands. Those signal waveforms are arranged from low frequency to high frequency as $C_{1}(t), C_{2}(t) \ldots C_{8}(t)$ shown in Fig. 6 . Then the energy of the eight reconstructed waveforms is extracted according to (3) in Fig. 7. Three principle frequency sub-bands which include the most dominant fault information are chosen and the correspond-ing entropies are calculated. Fig. 8 shows the entropy sequences of the three selected waveforms of Bearing 1-3. The entropy sequence is decomposed by EMD to obtain 11 IMFs as shown in Fig. 9. By accumulating the last four IMFs using (9), the sequence trend is

Table 1

Bearing operation conditions.

\begin{tabular}{|c|c|c|c|c|}
\hline Dataset & $\begin{array}{l}\text { Load } \\
(N)\end{array}$ & $\begin{array}{l}\text { Speed } \\
\text { (rev/min) }\end{array}$ & Training data & Testing data \\
\hline Dataset 1 & 4000 & 1800 & $\begin{array}{l}\text { Bearing 1-1 } \\
\text { Bearing 1-2 }\end{array}$ & $\begin{array}{l}\text { Bearing 1-3 Bearing 1-4 } \\
\text { Bearing 1-5 Bearing 1-6 } \\
\text { Bearing 1-7 }\end{array}$ \\
\hline Dataset 2 & 4200 & 1650 & $\begin{array}{l}\text { Bearing 2-1 } \\
\text { Bearing 2-2 }\end{array}$ & $\begin{array}{l}\text { Bearing 2-3 Bearing 2-4 } \\
\text { Bearing 2-5 Bearing 2-6 } \\
\text { Bearing 2-7 }\end{array}$ \\
\hline Dataset 3 & 5000 & 1500 & Bearing 2-1 & Bearing 2-2 Bearing 2-3 \\
\hline
\end{tabular}

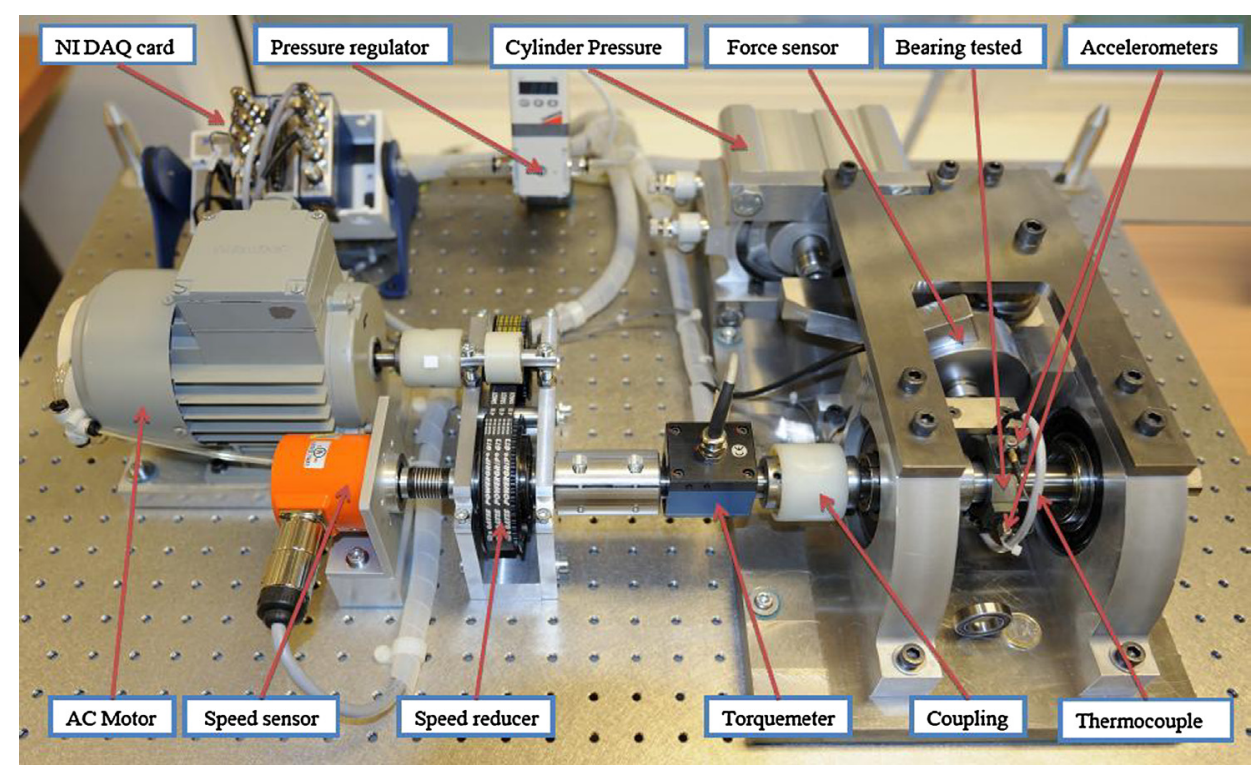

Fig. 3. Overview of the experimentation platform. 
extracted shown in Fig. 9. And then feature vectors of the selected sub-bands are composited using (10).

The former one hundred feature vectors of each bearing, which represent the normal state, are used for training the SOM. After

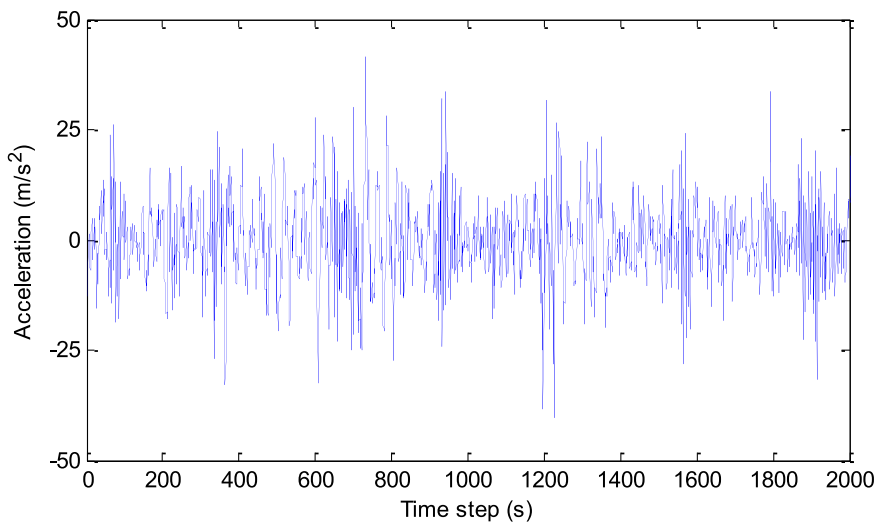

Fig. 4. The vibration acceleration signal of Bearing 1-3.
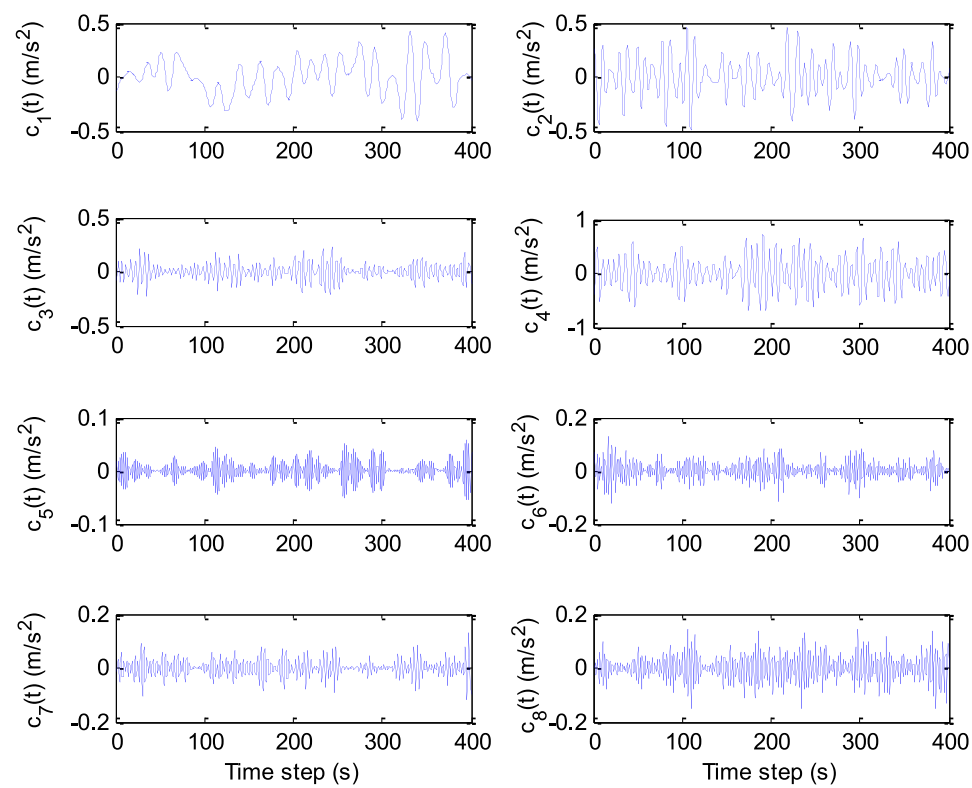

Fig. 6. The decomposition results of bearing vibration signal by WPD.
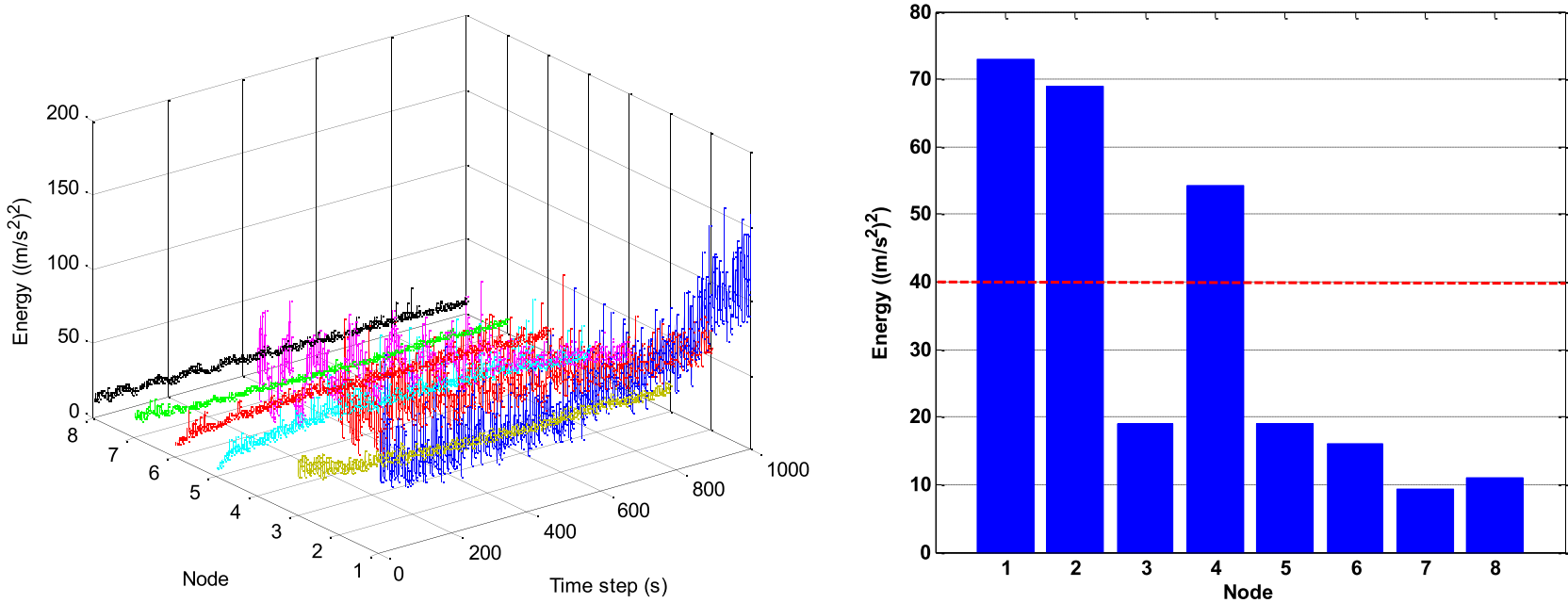

Fig. 7. Energy of each wavelet packet sub-band. 


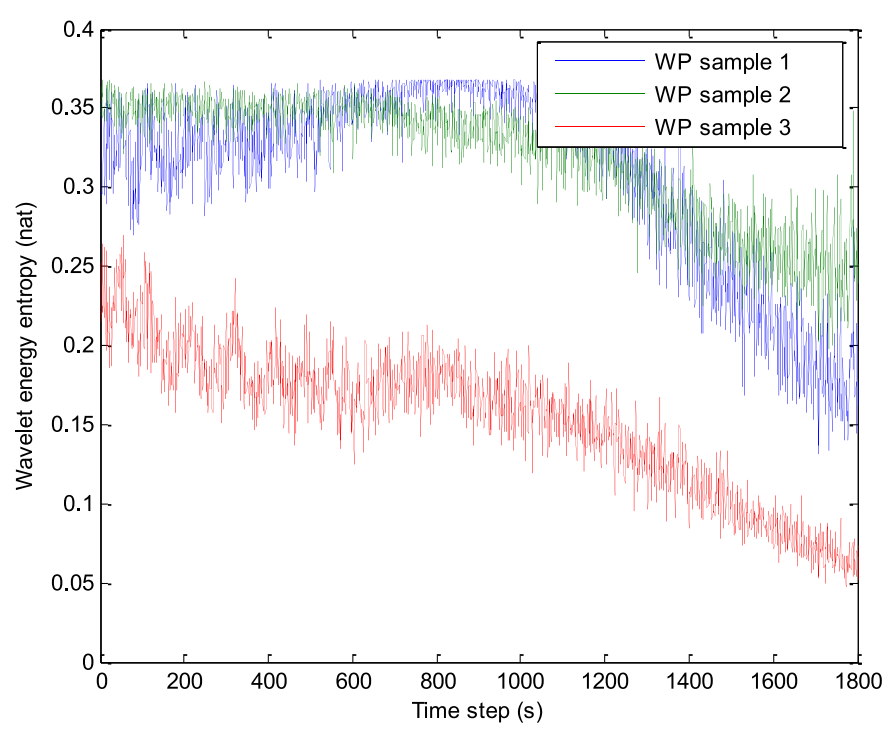

Fig. 8. Three signal entropies of the wavelet packet sub-bands.

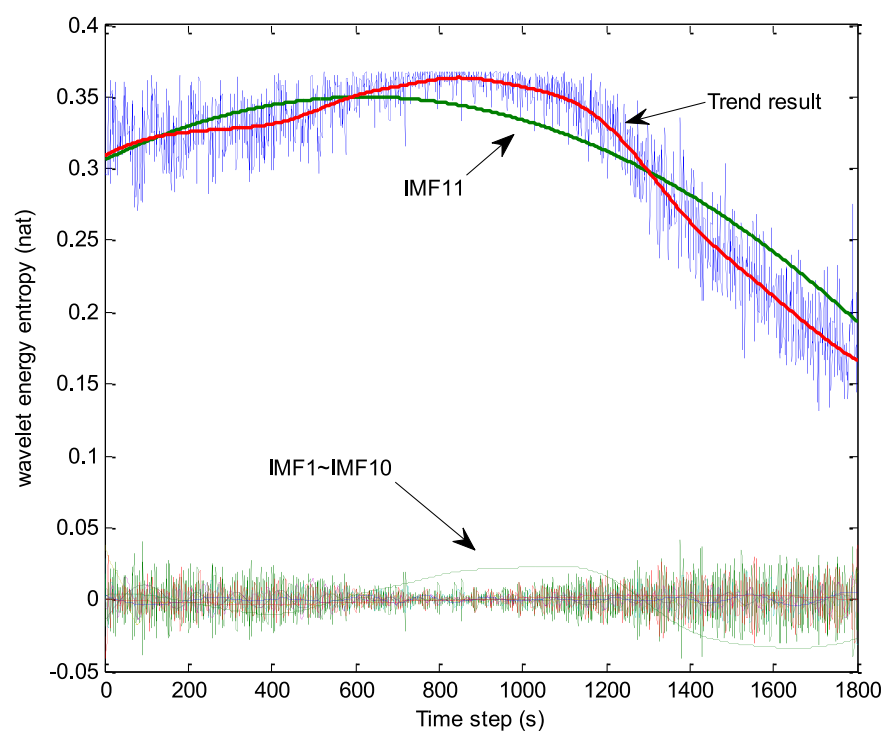

Fig. 9. The decomposition results of wavelet packet entropy by EMD.

training, all testing signals are used for testing and the corresponding CV curves of dataset 1 are shown in Fig. 10. Each stage's threshold can be set according to real situation. By the contrast analysis, we can see the CVs in normal state are high, whereas those at the ending stage of the experiment are relatively low.

Previous studies have reported that multiple faults including inner race fault, outer race fault, ball fault, cage fault, are found at the end of the experiment. Compared with those features shown in Fig. 5, we can find that the CV curves not only can obviously reflect the occurrence of early degradation of different faults, but also can effectively demonstrate the whole stage of degradation development. Assessment and maintenance strategy can be taken based on the CV curves and the state of the bearing.

\subsection{RUL estimation and analysis}

Since the CV curves have been obtained, the RUL of the bearing can be estimated using regression algorithm. Gaussian Process Regression (GPR) algorithm is applied to predict the RUL. GPR is a promising kernel machine-learning technique and has received increased attention over the past years [31]. It provides a good

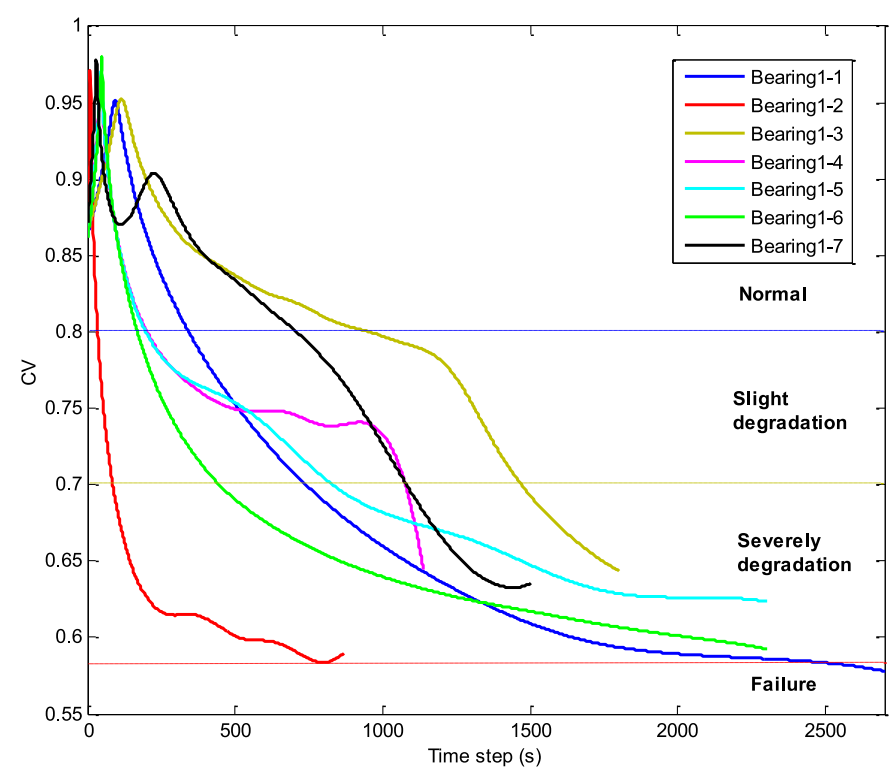

Fig. 10. Condition assessments of the testing bearing datasets using CV.

Table 2

Comparison between RUL prediction and Actual RUL.

\begin{tabular}{lrrrrr}
\hline $\begin{array}{l}\text { Test bearing } \\
\text { ID }\end{array}$ & $\begin{array}{l}\text { Current } \\
\text { life (s) }\end{array}$ & $\begin{array}{l}\text { Actual } \\
\text { RUL (s) }\end{array}$ & $\begin{array}{l}\text { RUL } \\
\text { prediction }(\mathrm{s})\end{array}$ & \multicolumn{1}{c}{$\begin{array}{l}\text { Er } \\
(\%)\end{array}$} & $\begin{array}{l}\text { Er 2 } \\
(\%)\end{array}$ \\
\hline Bearing1_3 & 18010 & 5730 & 5790 & -1.04 & 37 \\
Bearing1_4 & 11380 & 339 & 410 & -20.94 & 80 \\
Bearing1_5 & 23010 & 1610 & 6090 & -278.26 & 9 \\
Bearing1_6 & 23010 & 1460 & 1180 & 19.18 & -5 \\
Bearing1_7 & 15010 & 7570 & 8110 & -7.13 & -2 \\
Bearing233 & 12010 & 7530 & 6740 & 10.49 & 64 \\
Bearing24 & 6110 & 1390 & 670 & 51.80 & 10 \\
Bearing2_5 & 20010 & 3090 & 2200 & 28.80 & -440 \\
Bearing2_6 & 5710 & 1290 & 1560 & -20.93 & 49 \\
Bearing2_7 & 1710 & 580 & 320 & 44.83 & -317 \\
Bearing3_3 & 3510 & 820 & 850 & -3.66 & 90 \\
\hline
\end{tabular}

adaptability to handle high dimensionality, small sample size and nonlinear problems. The squared exponential (SE) covariance function is selected and the hyper-parameters in the SE covariance function are optimized by maximizing the marginal likelihood algorithm.

We use the CV curve data as input vector and calculate the output prediction. The RUL of the bearing can be obtained based on the predicted values and final threshold. Due to different working conditions, the failure thresholds, according to the learning data of each dataset, are set as 0.57 for dataset 1, 0.7 for dataset 2 and 0.68 for dataset 3. CV curves are then used to estimate the RUL of each bearing.

The estimation result of the RUL is depicted in Table 2. For each testing bearing, the prediction is made at the end of training data (see in column 2), and the corresponding error rate is defined as follows,

$E r=\frac{\operatorname{ActRUL}_{i}-\text { PredRUL }_{i}}{\operatorname{ActRUL}_{i}} \times 100 \%$

where $A c t R U L_{i}$ is the actual RUL of $i$-th bearing in column 3 and PredRUL $_{i}$ is the RUL prediction using the proposed method in column 4. For most test bearings, the predictions are feasible. A major deviation is found in Bearing 1-5, whose CV curve doesn't reach the failure threshold of dataset 1 until $29100 \mathrm{~s}$ in simulation. One reason is the lack of learning data. If more learning data were provided for training the model, the RUL results would have performed much better. Another cause of the abnormal results is the 
multiple faults may introduce a lot of uncertainty to the predictions. In order to simplify the assessment model, we make a trade off in the proposed method in terms of the precision and the complexity, which may leads to the large deviation.

A similar study, which is a winner solution strategy of 2012 challenge competition, has been published using the same bearing data [32]. The prediction percent error result is present in column 6 . The author describes a methodology for bearing RUL estimating using anomaly detection, degradation feature extrapolation and survival time ratios. That model works perfectly for some test bearing, but a larger deviation occurred as well. Compared with that model, 7 out of 11 bearings have less estimate percent error using the method proposed in this paper. According to the scoring method in [30], the score of the proposed method is 0.4181 and the score of that model is 0.3535 using (14) and (15), which means by using the prognostic model in the paper, the prediction accuracy can be increased by $5 \%$ for an early prediction.

$A_{i}= \begin{cases}\exp ^{-\ln (0.5) \cdot\left(E r_{i} / 5\right)} & \text { if } E r_{i} \leqslant 0 \\ \exp ^{+\ln (0.5) \cdot\left(E r_{i} / 20\right)} & \text { if } E r_{i}>0\end{cases}$

Score $=\frac{1}{11} \sum_{i=1}^{11} A_{i}$

\section{Conclusion}

This paper presents an assessment method for evaluating the state of the bearing's degradation and estimating the RUL. Multiple widely used techniques, such as WPD, EMD and SOM neural network, are applied as a combination method. The wavelet packet energy entropy supplies an effective way to reflect the change of degradation and EMD provides a simple but powerful way for extract waveform trend. Thus, we design a health indicator named $\mathrm{CV}$ to reflect the health state and propose a new method for the bearing degradation assessment based on those techniques. From the experiment result and the comparison, it can be concluded as follows:

(1) The combination of feature extraction method based on WPEMD successfully reflects the degradation using wavelet packet energy entropy than those traditional features, such as RMS, kurtosis and Crest factor.

(2) The CV derived from the SOM network can effectively identify the current degradation stage, which will help to realize the equipment condition based maintenance.

(3) The proposed method provides a useful tool for RUL estimation and the prediction accuracy is acceptable for the realword scenario.

\section{Acknowledgments}

The authors are highly thankful for the financial support of National Program on Key Basic Research Program of China under grant No. 2014CB744904, National Natural Science Foundation of China under grant No. 61304111 and Fundamental Research Funds for the Central Universities under grant No. YWF-13-JQCJ, China.

\section{References}

[1] A. Heng, S. Zhang, A.C. Tan, J. Mathew, Rotating machinery prognostics: State of the art, challenges and opportunities, Mech. Syst. Signal Process. 23 (2009) 724-739.

[2] C. Hu, B.D. Youn, P. Wang, J. Taek Yoon, Ensemble of data-driven prognostic algorithms for robust prediction of remaining useful life, Reliab. Eng. Syst. Saf. 103 (2012) 120-135.

[3] T. Wang, Bearing life prediction based on vibration signals: A case study and lessons learned, in: IEEE Conference on Prognostics and Health Management (PHM), IEEE, 2012, pp. 1-7.
[4] X. Si, W. Wang, C. Hu, D. Zhou, Remaining useful life estimation-A review on the statistical data driven approaches, European J. Oper. Res. 213 (2011) 1-14.

[5] G.F. Bin, J.J. Gao, X.J. Li, B.S. Dhillon, Early fault diagnosis of rotating machinery based on wavelet packets-Empirical mode decomposition feature extraction and neural network, Mech. Syst. Signal Process. 27 (2012) 696-711.

[6] T.L. Xu, X.Z. Lang, X.Y. Zhang, X.C. Pei, The research based on empirical mode decomposition in bearing fault diagnosis, Appl. Mech. Mater. 103 (2012) 225-228.

[7] J. Cheng, Y. Yang, Y. Yang, A rotating machinery fault diagnosis method based on local mean decomposition, Digit. Signal Process. 22 (2012) 356-366.

[8] P.K. Kankar, S.C. Sharma, S.P. Harsha, Fault diagnosis of ball bearings using con-tinuous wavelet transform, Appl. Soft Comput. 11 (2011) 2300-2312.

[9] L. Gao, Z. Yang, L. Cai, H. Wang, P. Chen, Roller bearing fault diagnosis based on nonlinear redundant lifting wavelet packet analysis, Sensors 11 (2010)260-277.

[10] Z. Shuai, Q. Yanhuai, H. Qingkai, Fault characteristics analysis for rotor systems with misalignment based on wavelet packet decomposition and frequency-band energy ratio analysis, in: 30th Chinese Control Conference (CCC), IEEE, 2011, pp. 4331-4335.

[11] F. Wang, Y. Zhang, B. Zhang, W. Su, Application of wavelet packet sample en-tropy in the forecast of rolling element bearing fault trend, in: International Conference on Multimedia and Signal Processing (CMSP), IEEE, 2011, pp. 12-16.

[12] Y. Lei, J. Lin, Z. He, M.J. Zuo, A review on empirical mode decomposition in fault diagnosis of rotating machinery, Mech. Syst. Signal Process. 35 (2013) 108-126.

[13] Y. Yu, C. Junsheng, A roller bearing fault diagnosis method based on EMD energy entropy and ANN, J. Sound Vib. 294 (2006) 269-277.

[14] C. Junsheng, Y. Dejie, Y. Yu, A fault diagnosis approach for roller bearings based on EMD method and AR model, Mech. Syst. Signal Process. 20 (2006) 350-362.

[15] A.R. Messina, V. Vittal, G.T. Heydt, T.J. Browne, Nonstationary approaches to trend identification and denoising of measured power system oscillations, IEEE Trans. Power Syst. 24 (2009) 1798-1807.

[16] F. Tavakkoli, M. Teshnehlab, A ball bearing fault diagnosis method based on wavelet and EMD energy entropy mean, in: International Conference on Intel-ligent and Advanced Systems, 2007, pp. 1210-1212.

[17] Z. Li, H. Gu, Y. Zhang, Fault diagnosis for rolling bearing based on wavelet packet and EMD, Coal Mine Mach. 6 (2010) 126.

[18] C. Wen, C. Zhou, The feature extraction of rolling bearing fault based on wavelet packet, EMD energy distribution, Appl. Mech. Mater. 233 (2012) 234-238.

[19] C. Wen, C. Zhou, The feature extraction of rolling bearing fault based on wavelet packet, empirical mode decomposition and kurtosis rule, in: Emerging Technologies for Information Systems Computing, and Management, in: Lect. Notes Electr. Eng., vol. 236, 2013, pp. 579-586.

[20] Q. Liu, F. Chen, Z. Zhou, Q. Wei, Fault diagnosis of rolling bearing based on wavelet package transform and Ensemble Empirical Mode Decomposition, Adv. Mech. Eng. 2013 (2013).

[21] A. Santhana Raj, N. Murali, Morlet wavelet UDWT denoising and EMD based bearing fault diagnosis, Electronics 17 (1) (2013).

[22] Y. Pan, J. Chen, X. Li, Bearing performance degradation assessment based on lifting wavelet packet decomposition and fuzzy c-means, Mech. Syst. Signal Process. 24 (2010) 559-566.

[23] Y. Pan, J. Chen, L. Guo, Robust bearing performance degradation assessment method based on improved wavelet packet-support vector data description, Mech. Syst. Signal Process. 23 (2009) 669-681.

[24] W. Caesarendra, A. Widodo, B. Yang, Application of relevance vector machine and logistic regression for machine degradation assessment, Mech. Syst. Signal Process. 24 (2010) 1161-1171.

[25] R. Huang, L. Xi, X. Li, C. Richard Liu, H. Qiu, J. Lee, Residual life predictions for ball bearings based on self-organizing map and back propagation neural network methods, Mech. Syst. Signal Process. 21 (2007) 193-207.

[26] Z. Wei, T. Tao, D. ZhuoShu, E. Zio, A dynamic particle filter-support vector regression method for reliability prediction, Reliab. Eng. Syst. Saf. 119 (2013) 109116.

[27] N.E. Huang, Z. Shen, S.R. Long, et al., The empirical mode decomposition and the Hilbert spectrum for nonlinear and non-stationary time series analysis, Proc. R. Soc. Lond. Ser. A Math. Phys. Eng. Sci. 454 (1998) 903-995.

[28] H. Qiu, J. Lee, J. Lin, G. Yu, Robust performance degradation assessment methods for enhanced rolling element bearing prognostics, Adv. Eng. Inform. 17 (2003) 127-140.

[29] J. Yu, A hybrid feature selection scheme and self-organizing map model for machine health assessment, Appl. Soft Comput. 11 (2011) 4041-4054.

[30] P. Nectoux, R. Gouriveau, K. Medjaher, E. Ramasso, B. Morello, N. Zerhouni, C. Varnier, PRONOSTIA: an experimental platform for bearings accelerated life test, in: IEEE International Conference on Prognostics and Health Management, Denver, CO, USA, 2012.

[31] C.E. Rasmussen, Gaussian processes in machine learning, in: Advanced Lectures on Machine Learning, 2004, pp. 63-71.

[32] E. Sutrisno, H. Oh, A.S.S. Vasan, M. Pecht, Estimation of remaining useful life of ball bearings using data driven methodologies, in: IEEE Conference on Prognostics and Health Management (PHM), IEEE, 2012, pp. 1-7. 
Sheng Hong was born in China, in 1981. He received his master degree and doctoral degree in communication and information system from Beihang University, in 2005 and 2009, respectively. He is now a graduate student advisor in the School of Reliability and System Engineering of Beihang University. His recent interests include signal processing, information system modeling, prognostics and heath management.

Zheng Zhou was born in China, in 1989. He was a graduate student in Beihang University for master degree. He is now with the systems engineering research institute, CSSC.

Enrico Zio is Director of the Chair in Complex Systems and the Energetic Challenge of Ecole Centrale Paris and Supelec, Director of the Grad- uate School of the Politecnico di Milano, Full Professor of Computational Methods for Safety and Risk Analysis, adjunct Professor in Risk Analysis at the University of Stavanger, Norway and at Universidad Santa Maria, Chile, and invited lecturer and committee member at various Master and $\mathrm{PhD}$ Programs in Italy and abroad.

Kan Hong was born in China, in 1982. He achieved his PhD degree in sensors from Cranfield University/UK Defence Academy. He is now a visiting scientist in the School of Reliability and System Engineering of Beihang University. His recent interests include spectral vision, machine learning, signal processing and image processing. 